Artigo original

Georgina Costa ${ }^{1}$

Sandra Afonso ${ }^{1}$

José Augusto Bragada ${ }^{1,2}$

Victor Machado Reis ${ }^{2.3}$

Tiago Manuel Barbosa ${ }^{1.2}$

\title{
ESTUDO COMPARATIVO DAS ADAPTAÇÕES FISIOLÓGICAS AGUDAS DURANTE A EXECUÇÃO DE TRÊS VARIANTES DE UM EXERCÍCIO BÁSICO DE HIDROGINÁSTICA
}

\author{
COMPARISON OF ACUTE PHYSIOLOGICAL ADAPTATIONS BETWEEN THREE \\ VARIANTS OF A BASIC HEAD-OUT WATER EXERCISE
}

\begin{abstract}
RESUMO
Foi objectivo do estudo comparar as adaptações fisiológicas agudas de variantes do mesmo exercício básico de Hidroginástica (acção exclusiva dos membros inferiores, acção simultânea dos membros inferiores e dos membros superiores, acção simultânea dos membros inferiores e dos membros superiores usando halteres flutuantes). Foram estudados 16 sujeito do sexo feminino, jovens, clinicamente saudáveis e com um nível de actividade física regular. Cada sujeito realizou, um exercício básico de Hidroginástica designado de "Cavalo-marinho". Antes e após cada execução de 6 minutos do exercício foi avaliada a percepção subjectiva de esforço (RPE) e a lactatemia ([La-]). Antes, durante e após cada execução foi avaliada a frequência cardíaca máxima atingida durante a exercitação (FCmax) e estimada a percentagem de frequência cardíaca máxima teórica atingida durante a exercitação (\%FCmax). Os sujeitos percepcionaram um aumento significativo da RPE passando da exercitação exclusiva da acção dos membros inferiores, para a exercitação simultânea dos membros inferiores e dos membros superiores, assim como, para a acção a exercitação simultânea dos membros inferiores e dos membros superiores com os halteres. O esforço cardíaco (FCmax e \%FCmax) foi significativamente inferior ao realizar o exercício básico estudado apenas com acção dos membros inferiores do que nas outras variantes. $\mathrm{O}$ aumento de segmentos em acção e a exercitação com os halteres promoveu incrementos significativos da [La-]. Concluindo, o incremento do número de segmentos em acção simultânea, assim como, a utilização de halteres flutuantes tendem a aumentar significativamente a resposta fisiológica aguda em Hidroginástica.
\end{abstract}

Palavras-chave: Hidroginástica; Variantes; Percepção subjectiva de esforço; Frequência cardíaca; Lactatemia.

\begin{abstract}
The aim of this investigation was to compare the acute physiological adaptations to several variants of the same basic head-out aquatic exercise (only with legs actions, with simultaneous legs and arms actions, with simultaneous legs and arms actions using buoyancy dumb-bells). 16 young females, clinically healthy and with a regular level of physical activity were studied. Each subjected performed a basic head-out aquatic exercise named "rocking horse". Before and after each 6 minutes exercise, rate of perceived exertion (RPE) and blood lactate (La']) were evaluated. Before, during and after each exercise, the maximal heart rate achieved (FCmax) was measured and the percentage of maximal theoretical heart rate estimated (\%FCmax). The subjects perceived an increasing exertion from the exercise only with legs actions to the exercise with simultaneous legs and arms actions, to the exercise with simultaneous legs and arms actions including dumb-bells. The cardiac workout (FCmax and \%FCmax) was significantly lower performing the exercise only with the legs than in the other two exercise conditions. The increasing number of limb's actions and the adoption of dumb-bells promoted an increase of the blood lactate. In conclusion, the increasing number of simultaneous limb's actions and the inclusion of materials, just like buoyancy dumb-bells, increased the acute physiological response in head-out aquatic exercises.
\end{abstract}

Key words: Head-out aquatic exercises; Exercise variants; RPE; Heart rate; Blood lactate.

1. Instituto Politécnico de Bragança, Departamento de Ciências do Desporto. Bragança, Portugal

2. Universidade de Trás-os-Montes e Alto Douro, Departamento de Desporto. Vila Real, Portugal

3. Saúde e Desenvolvimento Humano. Centro de Investigação em Desporto. Vila Real, Portugal.. 


\section{INTRODUÇÃO}

De entre as actividades físicas orientadas para a prevenção primária da saúde (vulgarmente denominadas de "actividades de fitness"), as actividades aquáticas tiveram uma forte expansão na última década. A Hidroginástica é um caso paradigmático, dado o elevado número de novos praticantes que aderem anualmente. Este aumento de adesões parece dever-se aos diversos benefícios de carácter fisiológico, biomecânico e psicológico recorrentemente atribuídos à actividade.

Nas sessões de Hidroginástica é frequente a realização de diversas variantes de um mesmo exercício. Com efeito, é vulgar na literatura técnica a descrição de um dado exercício realizando': (i) exclusivamente a acção dos membros inferiores; (ii) simultaneamente a acção dos membros inferiores e dos membros superiores e; (iii) simultaneamente a acções dos quatro membros, acrescido da utilização de materiais auxiliares. A adopção destas variantes tem em vista, diversificar a sessão e, aparentemente, prescrever aos alunos exercícios com níveis de intensidade ajustados às suas características individuais e níveis de aptidão física. Com efeito, esta também é uma estratégia metodológica adoptada nas actividades no meio terrestre, como por exemplo programas de Aeróbica ou de Dança ${ }^{2,3}$.

Existem diversas variáveis fisiológicas que são estudadas recorrentemente para avaliar, ou para estimar, a intensidade de exercitação das actividades físicas orientadas para a prevenção primária da saúde. Entre essas variáveis encontram-se a percepção subjectiva de esforço, a frequência cardíaca e a concentração sérica de lactato ${ }^{4,5}$.

A percepção subjectiva de esforço (RPE) é uma das variáveis psico-fisiológicas mais avaliadas nas actividades aquáticas, especialmente em Hidroginástica $^{6,7,8}$. Para o efeito é utilizada a escala mais recente de Borg ${ }^{9}$. De acordo com o American College of Sports Medicine ${ }^{10}$ para sujeitos jovens e clinicamente saudáveis, durante um programa de actividade física orientada para a saúde, a RPE deve variar entre os 14 e os 16 valores. Existem diversos grupos de investigação que estudaram o efeito da acção exclusiva dos membros superiores e dos membros inferiores na RPE ${ }^{11,12}$. Verificou-se uma RPE significativamente superior durante a realização de tarefas em cicloergómetros para os membros superiores do que para os membros inferiores ${ }^{11,12,13}$. Já Schaeffer-Gerschutz et al. ${ }^{14}$ ao analisarem a RPE após uma rotina de Aeróbica, verificaram que a percepção de esforço foi significativamente superior quando não se efectuava a acção dos membros superiores. Não obstante estes dados, não foi identificada na literatura qualquer investigação comparando a RPE de diversas variantes de exercícios básicos de Hidroginástica.

A frequência cardíaca (FC) é outra variável estudada recorrentemente neste tipo de actividades. O American College of Sports Medicine ${ }^{10}$ sugere, para sujeitos jovens e clinicamente saudáveis, actividades que promovam esforços cardíacos entre os $60 \%$ e os $90 \%$ do valor máximo. Apenas um trabalho comparou as adaptações fisiológicas agudas entre diversas variantes de um mesmo exercício básico de Hidroginástica ${ }^{15}$. Nesse estudo, verificou-se que a FC foi significativamente superior durante a execução de exercícios com materiais auxiliares do que sem a sua utilização. Já comparando o mesmo exercício aquático em função das acções segmentares realizadas, Darby e Yaeckle ${ }^{3}$ constataram que a FC foi significativamente superior quando realizada a acção simultânea dos quatro membros do que apenas dos membros inferiores. No meio terrestre, Butts et al. ${ }^{13}$ ao compararem, em tapete rolante, a caminhada normal e a caminhada incorporando um exercício específico para os membros superiores, verificaram um aumento significativo da FC para a segunda condição.

As actividades físicas orientadas para a prevenção primária da saúde apresentam características eminentemente aeróbias, pelo que a concentração sérica de lactato ([La-]) durante e após a sua prática não deve exceder o valor associado ao limiar anaeróbio (4 mmol. $\mathrm{I}^{-1}$ ). O American College of Sports Medicine ${ }^{10}$ sugere suas linhas orientadoras a realização de actividades de intensidade moderada. Ou seja, propõe a prática de actividades abaixo do limiar anaeróbio. No único estudo sobre este assunto, Borg et al. ${ }^{11}$ constataram, terrestre, que a [La'] foi significativamente superior durante a prática de actividades com os membros superiores do que com a acção dos membros inferiores. Todavia, não existe publicado na literatura qualquer estudo comparando a [ $\left.\mathrm{La}^{-}\right]$entre diferentes variantes de um determinado exercício de Hidroginástica.

Em síntese, ao contrário do meio terrestre ${ }^{2,16}$ a quantidade de estudos empíricos, procurando conhecer as reais repercussões fisiológicas de diferentes variantes do mesmo exercício de Hidroginástica estão longe de serem os desejáveis para uma cabal compreensão do fenómeno e um maior rigor na sua prescrição.

Grande parte da literatura sobre as adaptações fisiológicas agudas em Hidroginástica centra a sua atenção no estudo de populações especiais ${ }^{8,17,18,19,20}$. Estudos tendo como objecto de análise sujeitos clinicamente saudáveis, ou pelo menos, não pertencentes às ditas populações especiais, são bastante mais reduzidos ${ }^{21,22}$.

Foi objectivo deste estudo comparar as adaptações fisiológicas agudas de diversas variantes do mesmo exercício básico de Hidroginástica em sujeitos jovens e clinicamente saudáveis.

\section{PROCEDIMENTOS METODOLÓGICOS}

\section{Amostra}

A amostra foi constituída por 16 sujeitos do sexo feminino (não grávidas, clinicamente saudáveis, fisicamente activas, alunas de um curso de graduação em Ciências do Desporto e com pelo menos um ano de participação em programas de Hidroginástica). Nenhum elemento da amostra apresentou qualquer 
patologia ortopédica ou musculo-esquelética nos 6 meses anteriores à recolha dos dados. A tabela 1 apresenta as principais características da amostra. Os procedimentos utilizados para a consecução deste trabalho respeitaram as normas internacionais de experimentação com humanos (p.e., a Declaração de Helsínquia de 1975) e foi aprovado pelo Comité de Ética do Instituto Politécnico de Bragança (Portugal) já que cumpre com a legislação nacional e internacional.

Tabela 1. Características da amostra.

\begin{tabular}{lc}
\hline & $(\mathrm{n}=16)$ \\
\hline Idade (anos) & $23.3 \pm 2.4$ \\
Estatura $(\mathrm{m})$ & $1.60 \pm 0.07$ \\
Massa corporal $(\mathrm{kg})$ & $55.8 \pm 5.4$ \\
Índice de massa corporal & $21.9 \pm 1.7$ \\
$\left(\mathrm{~kg} \cdot \mathrm{m}^{-2}\right)$ & \\
$\begin{array}{l}\text { Actividade física (min sema- } \\
\text { na-1) }^{-1}\end{array}$ & $387.5 \pm 219.3$ \\
\hline
\end{tabular}

\section{Protocolo Experimental}

Durante 6 minutos, cada sujeito realizou o exercício básico de Hidroginástica "Cavalo-marinho", em imersão até ao nível do apêndice xifóide. O exercício foi efectuado a um ritmo musical de 136 batimentos minuto $^{-1}(2.26 \mathrm{~Hz})$ e ao ritmo de execução "tempo de água". O "Cavalo-marinho" tem como objectivo o desenvolvimento da capacidade cardio-respiratória. O Cavalo-Marinho é um exercício realizado com o joelho de um dos membros inferiores flectido e elevado. Troca-se o apoio, ficando com o membro inferior que estava a suportar o peso do sujeito suspenso atrás e em hiperextensão. Os membros superiores encontram-se estendidos efectuando uma adução ou uma abdução horizontal durante a troca dos apoios. A figura 1 apresenta uma ilustração do exercício básico estudado. Foram adoptadas três condições de exercitação: (i) com acção exclusiva dos membros inferiores, mantendo as mãos apoiadas ao nível da cintura pélvica; (ii) com acção simultânea da acção dos membros inferiores e dos membros superiores e; (iii) com acção simultânea das acções dos quatro membros, segurando halteres flutuantes (Halter-Gym H-907, Golfinho Sports, Coimbra, Portugal) com os seus eixos longitudinais orientados perpendicularmente aos eixos longitudinais dos antebraços. Foi adoptada uma ordem aleatória para a execução das três condições de exercitação, entre os diferentes sujeitos da amostra.

Cada exercício foi realizado continuamente durante 6 minutos de forma a permitir uma estabilização dos parâmetros fisiológicos estudados ${ }^{5,21}$. O ritmo musical foi adoptado de acordo com as recomendações da literatura técnica de Hidroginástica ${ }^{1}$, sendo o ritmo utilizado recorrentemente neste tipo de programas. $O$ ritmo "tempo de água" caracteriza-se pela contagem de um batimento musical em cada dois batimentos do ritmo da música ${ }^{1}$. Durante a primeira batida, fazse o apoio do membro inferior direito e inicia-se a adução horizontal dos membros superiores. Durante a segunda batida, o sujeito finaliza ambas as acções segmentares. Durante a terceira batida musical, os membros superiores iniciam a abdução horizontal e o apoio do membro inferior esquerdo. Na quarta batida, o sujeito finaliza as acções segmentares. Em todas as condições de exercitação a frequência de execução do movimento foi de $1.13 \mathrm{~Hz}$.

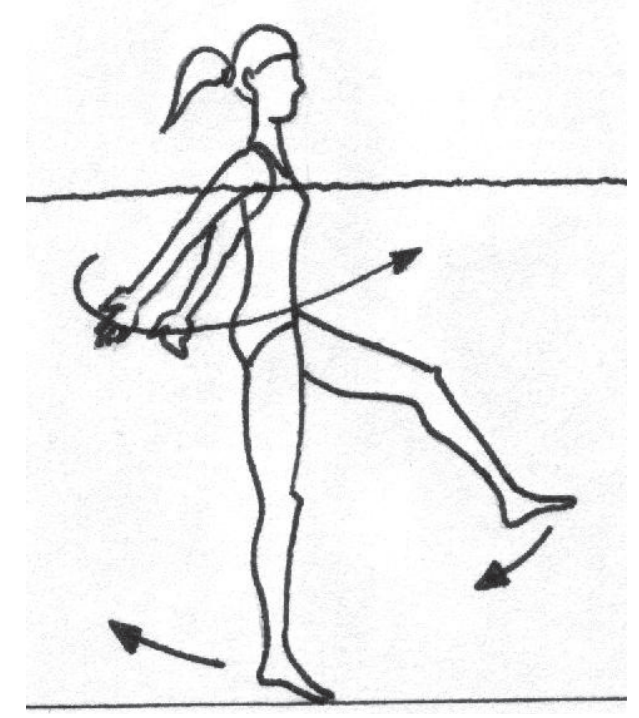

Figura 1. Ilustração do exercício básico de Hidroginástica "Cavalo-marinho".

Entre cada condição de exercitação os sujeitos tiveram pelo menos 60 minutos de recuperação. Este intervalo foi adoptado para permitir a remoção do lactato sanguíneo acumulado ${ }^{23}$. A água estava a uma temperatura de $29^{\circ} \mathrm{C}$, o ar ambiente a $31^{\circ} \mathrm{C}$ e a umidade relativa era de $60 \%$.

\section{Coleta de dados}

Antes e imediatamente após cada execução de 6 minutos foi avaliada a RPE, através da mais recente escala de Borg 6-209,24. No final da tarefa foi apresentada a cada sujeito uma folha com a escala e solicitava-se que ele indicasse o valor mais próximo do esforço geral que percepcionou para a condição de exercitação. Nesta escala a RPE varia entre 6 (nenhum esforço) e 20 (esforço máximo).

Antes, durante e após cada execução foi medida a FC através de um cardiofrequencímetro (Vantage NV, Polar, Kempele, Finlândia). A FC foi registada em intervalos de 5 segundos. Foi avaliada a frequência cardíaca máxima atingida durante a exercitação (FCmax). A percentagem de frequência cardíaca máxima teórica atingida durante a exercitação (\%FCmax) foi estimada através do procedimento sugerido por Wilmore e Costill ${ }^{5}$ em que:

$\% F C \max =\frac{F C \max }{220-\text { idade }} \times 100$

A [La'] foi avaliada a partir de um analisador de lactato (YSI 1500, Yellow Springs, Ohio, EUA) através 
da col de $25 \mu$ l de amostras de sangue capilar do lóbulo da orelha. Foram efectuadas col antes, imediatamente após o final da tarefa, assim como, aos 1, 3, 5 e 7 minutos de recuperação. Foi considerado para o efeito o valor máximo da [La-] observada.

\section{Estatística}

No que diz respeito à análise exploratória e descritiva, foram analisados os parâmetros de tendência central e de dispersão (média $\pm 1 \mathrm{DP}$ ) de todas as variáveis dependentes. A normalidade da distribuição foi estudada através do teste de Kolmogorov-Smirnov. Relativamente à análise inferencial, para estudar o efeito das diferentes condições de exercitação nas variáveis dependentes, calcularam-se ANOVA medidas repetidas (comparação intra-individual). Em todos os procedimentos foi adotado um nível de significância em que $P \leq 0.05$. A análise estatística foi efectuada num programa informático específico (SPSS, versão 9.0, Chicago, Illinois, EUA).

\section{RESULTADOS}

A Figura 2 apresenta a comparação das adaptações fisiológicas agudas, nas diferentes condições de exercitação, do exercício básico de Hidroginástica "Cavalo-marinho".

A RPE apresentou uma interacção significativa com a condição de exercitação $[F(2,15)=146.96$; $P$ <
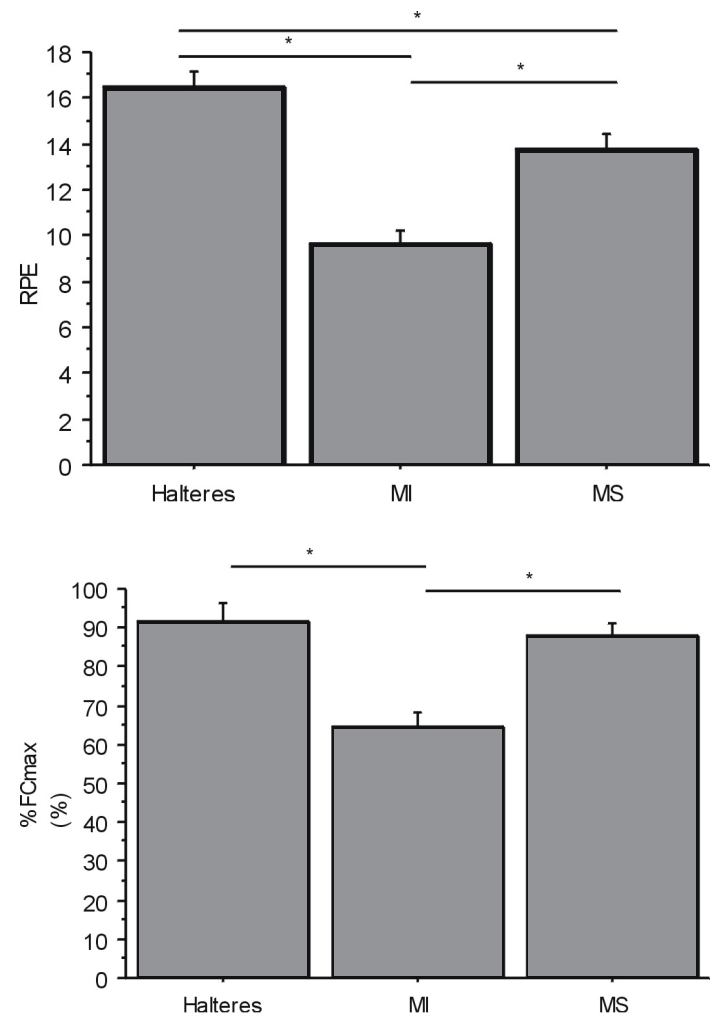

${ }^{*} \mathrm{P} \leq 0.05$ de Hidroginástica "Cavalo-marinho".
0.01]. As diferenças da RPE ocorreram entre as três variantes do exercício em estudo. Comparando os pares de condições de exercitação, constatou-se a existência de diferenças significativas entre os valores médios da acção exclusiva dos membros inferiores com a acção simultânea dos membros inferiores e dos membros superiores $(P<0.01)$, entre os valores médios da acção exclusiva dos membros inferiores com a acção simultânea dos membros inferiores e dos membros superiores usando halteres $(P<0.01)$ e entre os valores médios da acção simultânea dos membros inferiores e dos membros superiores com a acção simultânea dos membros inferiores e dos membros superiores usando halteres $(P<0.01)$. Isto é, os sujeitos percepcionaram um aumento significativo do esforço passando da exercitação exclusivamente da acção dos membros inferiores, para a exercitação simultânea dos membros inferiores e dos membros superiores, assim como, para a acção a exercitação simultânea dos membros inferiores e dos membros superiores usando os halteres.

Os dois parâmetros associados ao esforço cardiovascular estudados tiveram um comportamento semelhante. A FCmax $[F(2,15)=50.97 ; P<0.01]$ e a $\% F C m a x ~[F(2,15)=89.58 ; P<0.01]$ apresentaram interacções significativas com as variante do exercício básico realizadas. Da comparação dos pares de condições de exercitação verificou-se a existência de diferenças significativas entre os valores médios da exercitação exclusivamente com a acção dos membros
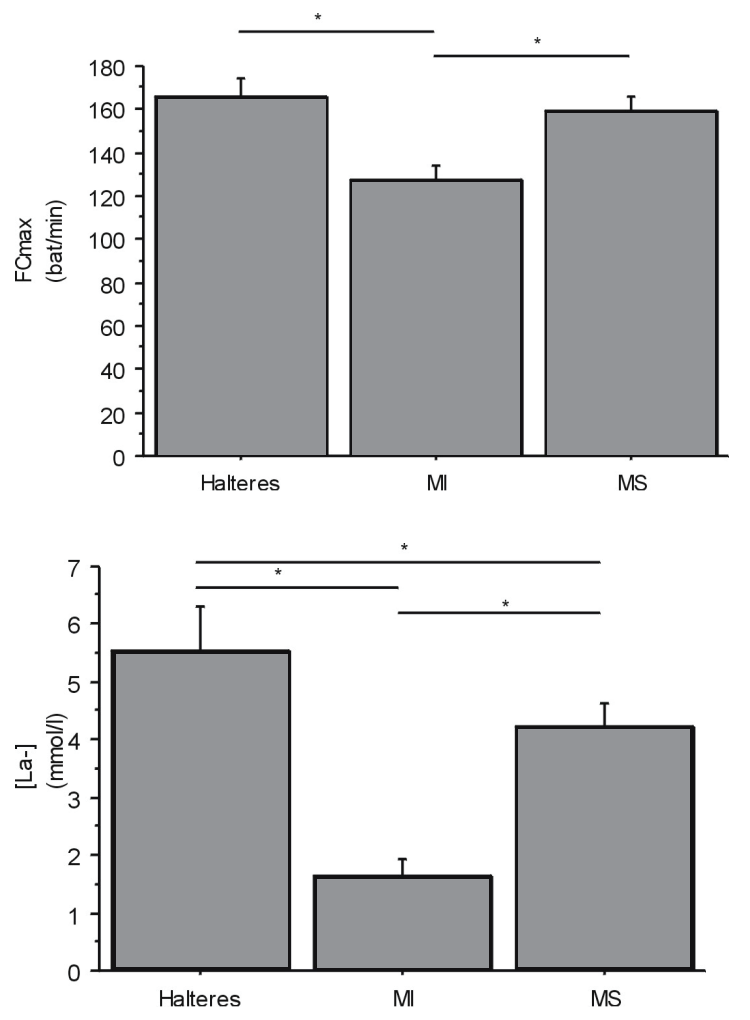

Figura 2. Comparação das adaptações fisiológicas agudas nas diferentes condições de exercitação do exercício básico 
inferiores e a acção simultânea dos membros inferiores e dos membros superiores $(P<0.01)$; assim como, entre os valores médios da exercitação exclusivamente com a acção dos membros inferiores e a acção simultânea dos membros inferiores e dos membros superiores usando halteres $(P<0.01)$ nos dois parâmetros. Ou seja, o esforço cardíaco foi significativamente inferior ao realizar o exercício básico estudado apenas com acção dos membros inferiores do que nas outras duas variantes.

No que se refere à [La-], este parâmetro também apresentou uma interacção significativa com a condição de exercitação $[\mathrm{F}(2,15)=83.14$; $\mathrm{P}<0.01]$. Comparando os pares de condições de exercitação constatou-se a existência de diferenças significativas entre todos os pares. O valor médio da [La'] com acção exclusiva dos membros inferiores foi significativamente inferior acção simultânea dos membros inferiores e dos membros superiores $(P<0.01)$. O valor médio da [La'] com a acção exclusiva dos membros inferiores foi significativamente inferior a acção simultânea dos membros inferiores e dos membros superiores usando halteres $(P<0.01)$. Finalmente, o valor médio da [La-] foi significativamente inferior durante a exercitação com acção simultânea dos membros inferiores e dos membros superiores acção simultânea dos membros inferiores e dos membros superiores usando halteres $(P<0.01)$.

\section{DISCUSSÃO}

Foi objectivo deste estudo comparar as adaptações fisiológicas agudas de diversas variantes do mesmo exercício básico de Hidroginástica (com acção exclusiva dos membros inferiores, com acção simultânea dos membros inferiores e dos membros superiores, com acção simultânea dos membros inferiores e dos membros superiores usando halteres flutuantes). A principal conclusão deste estudo foi que o incremento do número de segmentos em acção simultânea, assim como, a utilização de halteres flutuantes tendem a aumentar significativamente a resposta fisiológica aguda em Hidroginástica.

O American College of Sports Medicine ${ }^{10}$, nas suas linhas orientadoras, sugere para sujeitos jovens e clinicamente saudáveis uma RPE entre os 14 e os 16 valores durante um programa de actividade física orientada para a saúde. Analisando-se os valores médios da RPE para as três condições de exercitação, constatou-se que a execução do movimento com acção simultânea dos membros inferiores e dos membros superiores, bem como, com a acção simultânea dos membros inferiores e dos membros superiores usando halteres estiveram dentro dos limites inferior e superior sugeridos pela dita organização. A RPE apresentou uma interacção significativa com a condição de exercitação. Os sujeitos percepcionaram um aumento significativo do esforço passando da exercitação exclusivamente da acção dos membros inferiores, para a exercitação simultânea dos membros inferiores e dos membros superiores, assim como, para a exercitação simultânea dos membros inferiores e dos membros superiores usando halteres flutuantes. O trabalho mecânico suplementar que o sujeito têm de realizar para vencer o arrasto hidrodinâmico, ao incrementar o número de segmentos em acção, promoverá uma maior percepção do esforço ${ }^{25,26}$. além de terem de vencer a resistência oferecida pela água durante a exercitação com os membros inferiores e o tronco, tem de superar o maior arrasto imposto pela acção dos membros superiores e pelo uso dos halteres, o que provoca um trabalho mecânico interno adicional ${ }^{27}$.

O American College of Sports Medicine ${ }^{10}$ propõe para o desenvolvimento do condicionamento cardiorespiratório um esforço cardíaco entre o moderado e o forte $(60 \% \leq \% \mathrm{FCmax} \leq 90 \%)$, solicitando grandes grupos musculares. As três condições de exercitação apresentaram valores médios dentro do intervalo definido. A FCmax e a \%FCmax evidenciaram interacções significativas com as variantes do exercício básico realizadas. O esforço cardíaco foi significativamente inferior ao realizar o exercício básico estudado apenas com acção dos membros inferiores do que nas outras duas condições. Este mesmo fenómeno foi previamente observado no meio aquático ${ }^{15}$ e no meio terrestre ${ }^{3,13}$. A inclusão da acção dos membros superiores com ou sem halteres flutuantes promoverá um aumento da percentagem de tecido músculoesquelético total metabolicamente activos e, portanto, um maior aporte de oxigénio e nutrientes que se traduzirá num incremento do esforço cardíaco.

No meio aquático, outro factor com repercussões na FC é a temperatura corporal ${ }^{28,29}$. A velocidade de transferência de calor relaciona-se com o gradiente térmico e com a condutibilidade térmica do meio onde ocorre a troca. Com efeito, o meio aquático caracterizase por apresentar uma elevada condutibilidade, pelo que o corpo humano arrefecerá mais depressa do que noutros meios ${ }^{29}$. Contudo, à medida que a derme é aquecida, os vasos sanguíneos superficiais dilatamse e o suprimento sanguíneo periférico aumenta. Isto promoverá uma redistribuição sanguínea de modo a fornecer volume sanguíneo à periferia, como ocorrerá em condições de exercitação com a acção dos membros superiores com ou sem a utilização de halteres flutuantes. A necessidade de promover uma maior irrigação sanguínea na periferia terá como consequência o aumento da $\mathrm{FC}^{5}$.

Os valores médios da [La]] foram próximos das $4 \mathrm{mmol} \cdot{ }^{-1}$ para a condição de exercitação simultânea da acção dos membros inferiores e dos membros superiores e claramente superiores para a exercitação com acção simultânea dos membros inferiores e dos membros superiores usando halteres flutuantes. Ou seja, a utilização de materiais auxiliares parecem privilegiar vias anaeróbias para a re-síntese do ATP. A [ $\left.\mathrm{La}^{-}\right]$apresentou uma interacção significativa com a condição de exercitação. O aumento de segmentos em acção e a exercitação com os halteres promoveu incrementos significativos da [La-]. Borg et al..$^{11}$ já tinham verificado que a [La'] era significativamente superior durante a prática de actividades com os membros 
superiores acção dos membros inferiores, pelo menos, no meio terrestre. A prática de exercícios básicos de Hidroginástica sem a utilização de materiais auxiliares será um meio óptimo de promover o desenvolvimento de componentes da aptidão física associada ao estímulo do sistema oxidativo. Ao incluir os halteres flutuantes, parece que o sistema aeróbio não consegue suprir completamente as necessidades de re-síntese do ATP, pelo que o sistema anaeróbio intervirá de forma a fornecer a energia suplementar exigida.

Relativamente ao meio terrestre, existem vários estudos em que se analisa as repercussões fisiológicas da realização de uma determinada tarefa motora com um número diferente de segmentos em acção e/ ou a utilização de cargas externas. As tarefas mais estudadas são a caminhada e a corrida. Verificou-se que a locomoção com cargas nas mãos ou nos punhos, como por exemplo halteres, induziam aumentos significativos da RPE, da FC, do custo energético e do dispêndio energético para um mesmo trabalho mecânico externo ou velocidade de deslocamento ${ }^{16}$. Mais ainda, a introdução da carga externa induziu aumentos estatisticamente significativos da pressão arterial ${ }^{16}$. Logo, a utilização destes equipamentos no meio terrestre - e possivelmente no meio aquático - devem ser contra-indicados a determinadas populações, tais como idosos ou indivíduos com historial de patologia cardiovascular. Todavia, a extrapolação de tais especulações para o meio aquático deve ser feita de forma cautelosa, já que a amostra do presente estudo era jovem, clinicamente saudável e com hábitos regulares de actividade física.

\section{CONCLUSÃO}

A principal conclusão deste estudo foi que o incremento do número de segmentos em acção simultânea, assim como, a utilização de halteres flutuantes tendem a aumentar significativamente a resposta fisiológica aguda em Hidroginástica. Comparando a resposta fisiológica das três condições de exercitação em estudo com as sugestões do American College of Sports Medicine ${ }^{10}$, a prescrição de exercícios básicos de Hidroginástica, com a acção simultânea dos membros inferiores e dos membros superiores, é uma actividade adequada para o condicionamento cardio-respiratório. A utilização de halteres flutuantes durante o segmento aeróbio da sessão de Hidroginástica deve ser realizada apenas em sujeitos clinicamente saudáveis, com níveis de aptidão física bastante elevados e tomando em consideração o sistema bioenergético que desejam estimular.

\section{REFERÊNCIAS BIBLIOGRÁFICAS}

1. Kinder T, See J. Aqua Aerobics - A Scientific Approach. Dubuque, IA: Eddie Bowers Publishing; 1992.

2. Darby LA, Browder KD, Reeves BD. The effects of cadence, impact and step on physiological responses to aerobic dance exercise. Res Q Exerc 1995; 66:231-238.
3. Darby L, Yaeckle B. Physiological responses during two types of exercise performed on land and in water. J Sports Med Phys Fitness 2000; 40:303-311.

4. Benelli P, Ditroilo M, de Vito G. Physiological responses to fitness activities: a comparison between land-based and water aerobics exercise. J Strength Cond Res 2004; 18:719-722.

5. Wilmore J, Costill D. Physiology of Sport and Exercise. Champaign, IL: Human Kinetics; 1994.

6. Barker K, Dawes H, Hansford P, Shamley D. Perceived and measured levels of exertion of patients with chronic back pain exercising in a hydrotherapy pool. Arch Phys Med Rehab 2003; 84:1319-1323.

7. Melton-Rogers S, Hunter G, Walter J, Harrison P. Cardiorespiratory responses of patients with rheumatoid arthritis during bicycle riding and running in water. Phys Ther 1996; 76:1058-1065.

8. Shono T, Fujishima K, Hotta N, Ogaki T, Masumoto K. Cardiorespiratory response to low intensity walking in water and on land in elderly women. J Physiol Anthropol 2001; 20:269-274.

9. Borg G. Perceived Exertion and Pain Scales. Champaign, IL: Human Kinetics Publishers; 1998.

10. American College of Sports Medicine. ACSM's Guidelines for Exercise Testing and Prescription. Baltimore, Maryland: Lippincott Williams \& Wilkins; 2000.

11. Borg G, Hassmen P, Lagertrom M. Perceived exertion related to heart rate and blood lactate during arm and leg exercise. Eur J Appl Physiol 1987; 56:679-685.

12. Kang J, Chaloupka EC, Mastragelo MA, Angelucci J. Physiological responses to upper body exercise on an arm and a modified leg ergometer. Med Sci Sports Exerc 1999; 31:1453-1459.

13. Butts NK, Knox KM, Foley TS. Energy costs of walking on a dual-action treadmill in men and women. Med Sci Sports Exerc 1995; 27:121-125.

14. Schaeffer-Gerschutz SA, Darby LA, Browder KD. Differentiated ratings of perceived exertion and physiological responses during aerobic steps by impact/type of arm movement. Percept Mot Skills 2000; 90:457-471.

15. Robert J, Jones L, Bobo M. The physiologic response of exercising in the water and on land with and without the X1000 Walk'n Tone exercise belt. Res $Q$ Exerc 1996; 67:310-315.

16. Graves JE, Pollock ML, Montain SJ, Jackson AS, O'Keefe JM. The effect of hand-held weights on the physiological responses to walking exercise. Med Sci Sports Exerc 1987; 19:260-265.

17. Mannerkorpi K, Ahlmen M, Ekdahl C. Six and 24-month follow-up of pool exercise therapy and education for patients with fybromyalgia. Scand J Rheumatol 2002; 31:306-310.

18. McMurray M, Berry M, Katz V. Hemodynamics of pregnant women during immersion and exercise. Int J Sports Med 1989; 10:443-447.

19. Pechter U, Ots M, Mesikepp S, Zilmer K, Kullissar T, Vihalemm T, Maaroos J. Beneficial effects of waterbased exercise in patients with chronic kidney disease. Int J Rehabil Res 2003; 26:153-156.

20. Wyatt F, Milman S, Manske R, Deere R. The effects of aquatic and traditional exercise programs in persons with knee osteoarthritis. J Strength Cond Res 2001; 15:337-340.

21. Barbosa TM, Garrido MF, Bragada JA. Estudo comparativo das adaptações fisiológicas agudas durante a exercitação em imersão ao nível do apêndice xifóide e da articulação coxo-femoral. Motricidade 2006; 2:23-31. 
22. Hered S, Darby LA, Yaekle B. Comparison of physiological responses to comparable land and water exercises. Med Sci Sports Exerc 1997; 29:S16.

23. Wakayoshi K, Tatesada E, Ono K, Terada A, Ogita $F$. Blood lactate response to various combinations of swimming velocity and rest period of interval training. In: Keskinen KL, Komi P, Hollander AP, organizadores. Biomechanics and Medicine in Swimming VIII. Jyväskylä: Gummerus Printing; 1999. p. 401-406.

24. Borg, G. Perceived exertion. Exerc Sci Rev 1974; 2:131-153.

25. DeMaere J, Ruby C. Effects of deep water and treadmill running on oxygen uptake and energy expenditure in seasonally trained cross country runners. J Sports Med Phys Fitness 1997; 37:175-181.

26. Yu E, Kitagawa K, Mutoh Y, Miyashita M. Cardiorespiratory responses to walking in water. In: Miyashita M, Mutoh $\mathrm{Y}$, Anderson AB, organizadores. Medicine and Science in Aquatic Sports. Basel: Karger; 1994. p. 39-41.
27. Fawcett C. Principles of aquatic rehab: a new look at hydrotherapy. Sports Med 1994; 7:6-9.

28. Srámek P, Simeckova M, Jansky L, Savlikova J, Vybiral $\mathrm{S}$. Human physiological responses to immersion into water of different temperatures. Eur J Appl Physiol 2000; 81:436-442.

29. Datta A, Tipton M. Respiratory response to cold water immersion, neural pathways, interactions and clinical consequence awake and asleep. J Appl Physiol 2006; 100:2057-2064.

\section{Agradecimentos}

Este estudo foi parcialmente suportado pelo Programa de Apoio ao Financiamento no Desporto (PAFID) da Secretaria de Estado do Desporto de Portugal.

\section{Endereço para correspondência}

Tiago Barbosa

Recebido em 04/12/07

Departamento de Ciências do Desporto

Revisado em 18/02/08

Instituto Politécnico de Bragança

Aprovado em 13/03/08

Campus Sta. Apolónia, Apartado 1101

5301-856 Bragança - Portugal

E-mail: barbosa@ipb.pt 